\title{
CINÉTICA E MODELAGEM DA SECAGEM DE BANANA PRATA EM SOPRADOR DE LEITO FLUIDIZADO TIPO TÚNEL PRECEDIDA DE DESIDRATAÇÃO OSMÓTICA
}

\author{
J. A. da SILVA ${ }^{1}$, R. T. BAIA ${ }^{1}$, W. L. RODRIGUES ${ }^{1}$ \\ ${ }^{1}$ Universidade do Estado do Amapá, Engenharia Química \\ E-mail para contato: jessica-eng@hotmail.com
}

\begin{abstract}
RESUMO - A secagem de frutas é um método bastante usado para prolongar sua vida útil. Na região norte do Brasil há grande diversidade de frutas e de outras matérias primas que poderiam ser mais bem utilizadas, gerando renda para a população local e impulsionando a economia. Este trabalho tem como objetivo analisar a cinética de secagem de bananas da variedade prata, aplicar seis modelos matemáticos de secagem e verificar a influência do pré-tratamento osmótico para o processo global de secagem. As secagens foram realizadas através de convecção forçada em túnel de vento, com velocidade do ar de $1 \mathrm{~m} / \mathrm{s}$ e a temperatura de $68^{\circ} \mathrm{C}$ e sob velocidade do ar de $0,5 \mathrm{~m} / \mathrm{s}$ e a $78^{\circ} \mathrm{C}$. Antes da secagem convectiva, as amostras sofreram pré-tratamento osmótico em solução concentrada de sacarose a $60 \%(\mathrm{~m} / \mathrm{m})$ e $75 \%(\mathrm{~m} / \mathrm{m})$ por 90 minutos para preservar algumas características sensoriais do produto, como textura e cor. Analisou-se qual modelo matemático melhor se ajustou aos dados experimentais através da comparação dos valores de erro médio relativo, erro médio estimado e do coeficiente de determinação. As curvas obtidas estão em conformidade com os dados encontrados na literatura e indicam que a desidratação osmótica foi eficaz na remoção de umidade das amostras de banana, reduzindo o tempo de secagem convectiva. Observou-se que o modelo matemático que apresentou melhor ajuste foi o de Midilli e Kucuk.
\end{abstract}

\section{INTRODUÇÃO}

Um dos desafios do mercado de frutas é melhorar a eficiência do produtor rural no processo de comercialização de sua produção reduzindo as perdas pós-colheita, que podem chegar a 40\% nos países em desenvolvimento (Gomes et al., 2007).

A secagem convectiva é um processo antigo utilizado para preservar alimentos, no qual o sólido a ser seco é exposto a uma corrente de ar quente que flui continuamente e assim a umidade é removida (Souza Neto et al., 2005). A remoção de umidade provoca a diminuição da atividade de água do produto, inibindo o desenvolvimento de microrganismos e retardando deteriorações de origem físico-química, o que aumenta o tempo de prateleira do produto (Cano-Chauca et al., 2004).

Apesar dos aspectos positivos a secagem convectiva pode alterar as características sensoriais e o valor nutricional dos alimentos, a intensidade dessas alterações é dependente das condições utilizadas no processo de secagem e das características próprias de cada produto, para preservar parâmetros como a cor, sabor e aroma de frutas, que são sensíveis à 
secagem por convecção em ar aquecido, pode se realizar uma etapa prévia de desidratação osmótica (Queiroz et al., 2007).

Este trabalho tem como objetivo analisar a secagem de bananas da variedade prata, avaliar qual o melhor modelo matemático de secagem e verificar a influência do prétratamento osmótico para o processo global de secagem.

\section{MATERIAIS E METODOLOGIA}

Os experimentos de secagem osmótica foram realizados no laboratório de físicoquímica e os experimentos de secagem convectiva desenvolvidos no laboratório de materiais do Núcleo Tecnológico de Engenharia, ambos localizados na Universidade do Estado do Amapá.

Foram utilizadas bananas da variedade prata cultivadas na zona rural da cidade de Macapá, Estado do Amapá. As bananas selecionadas apresentavam estágio de maturação ideal para consumo, estágio VI de maturação, observando-se critérios de uniformidade do grau de maturação, integridade física e tamanho similar.

Os frutos foram lavados, sanitizados em solução com hipoclorito de sódio, descascados, raspados (para evitar o escurecimento durante as secagens) e fatiados em espessura de $5 \mathrm{~mm}$. O diâmetro médio das fatias foi $3 \mathrm{~cm}$.

Os frutos fatiados foram pesados, após a massa da amostra ser determinada, a mesma foi transferida para o béquer contendo a solução de sacarose, onde permaneceu por 1 hora e 30 minutos sob agitação constante a temperatura ambiente (aproximadamente $23^{\circ} \mathrm{C}$ ).

A solução de sacarose foi preparada com açúcar cristalizado granulado da marca Milla, obtido em comércio local, e água destilada. O volume de solução em cada béquer foi de 600 $\mathrm{mL}$ e a massa de açúcar de acordo com a proporção estabelecida para cada experimento: 360 gramas para a concentração de $60 \%(\mathrm{~m} / \mathrm{m})$ e 450 gramas para a concentração de $75 \%(\mathrm{~m} / \mathrm{m})$.

Os trabalhos encontrados na literatura, como Corrêa et al. (2008) e Marques et al. (2007), procederam a desidratação osmótica em solução de sacarose em concentração menor do que a escolhida neste trabalho, mas o tempo de contato era bem superior, no entanto, os resultados dos autores mostram que a taxa difusiva mais efetiva se dá na primeira hora e vai decrescendo. De maneira a otimizar este processo, consequentemente reduzindo o tempo, escolheram-se concentrações maiores.

Os experimentos de secagem convectiva foram realizados após o tratamento prévio por desidratação osmótica, exceto em dois experimentos. Esse procedimento foi adotado para avaliar se a desidratação osmótica apresenta relevância para a perda de água na secagem global.

As secagens convectivas foram realizadas sob duas condições, uma com temperatura de $68{ }^{\circ} \mathrm{C}$ e velocidade do ar de $1,0 \mathrm{~m} / \mathrm{s}$ e outra sob $78^{\circ} \mathrm{C}$ e $0,5 \mathrm{~m} / \mathrm{s}$, para avaliar a influência na secagem. A ordem e as condições dos experimentos são apresentadas na Tabela 1. 
Tabela 1 - Ordem e parâmetros dos experimentos

\begin{tabular}{c|c|c|c}
\hline Exp (n) & Concentração $(\% \mathbf{~ m} / \mathbf{m})$ & $\mathbf{T}\left({ }^{\circ} \mathbf{C}\right)$ & Velocidade do $\mathbf{a r}(\mathbf{m} / \mathbf{s})$ \\
\hline $\mathbf{1}$ & Sem tratamento & 68 & 1,0 \\
\hline $\mathbf{2}$ & Sem tratamento & 78 & 0,5 \\
\hline $\mathbf{3}$ & 60 & 68 & 1,0 \\
\hline $\mathbf{4}$ & 60 & 78 & 0,5 \\
\hline $\mathbf{5}$ & 75 & 68 & 1,0 \\
\hline $\mathbf{6}$ & 75 & 78 & 0,5 \\
\hline \multicolumn{2}{|r}{}
\end{tabular}

A amostra foi inserida no túnel de secagem, onde permaneceu até que a sua massa não variasse significativamente em três pesagens consecutivas. Essas pesagens foram realizadas regularmente, começando com 5 minutos de intervalo, depois aumentando gradativamente. Após a secagem convectiva, a amostra foi levada à estufa para dar continuidade ao processo de remoção de água e, assim, encontrar o valor da massa seca. Com os dados obtidos foi possível determinar os parâmetros para o estudo da cinética do processo.

Os modelos escolhidos para representação da umidade em função do tempo são apresentados na Tabela 2. O ajuste foi realizado no software Statistica ${ }^{\circledR} 7.0$, através de estimativa não linear e com o uso do método Quasi-Newton. Onde RU é razão de umidade do produto (adimensional); $\mathrm{t}$ é o tempo de secagem (min); $\mathrm{K}, \mathrm{K}_{0}$ e $\mathrm{K}_{1}$ são coeficientes de secagem; a, b, e n são constantes dos modelos.

Tabela 2 - Modelos matemáticos utilizados

\begin{tabular}{c|lr|l}
\hline \multicolumn{1}{|c|}{ Modelo } & \multicolumn{1}{|c}{ Equação } & \multicolumn{1}{|c}{ Fonte } \\
\hline Newton & $\mathrm{RU}=\exp (-\mathrm{k} \times \mathrm{t})$ & $(1)$ & $($ Lewis, 1921) \\
\hline Page & $\mathrm{RU}=\exp \left(-\mathrm{k} \times \mathrm{t}^{\mathrm{n}}\right)$ & $(2)$ & $($ Page, 1949) \\
\hline Midilli e Kucuk & $\mathrm{RU}=\mathrm{a} \times \exp \left(-\mathrm{k} \times t^{n}\right)+\mathrm{b} \times \mathrm{t}$ & $(3)$ & $($ Midilli et al.,2002) \\
\hline Aproximação da difusão & $\mathrm{RU}=\mathrm{a} \times \exp (-\mathrm{k} \times \mathrm{t})+(1-\mathrm{a}) \times \exp (-\mathrm{k} \times \mathrm{b} \times \mathrm{t})(4)$ & $($ Kassen, 1998) \\
\hline Henderson e Pabis & $\mathrm{RU}=\mathrm{a} \times \exp (-\mathrm{k} \times \mathrm{t})$ & $(5)$ & $($ Henderson e Pabis, 1961) \\
\hline Dois termos & $\mathrm{RU}=\mathrm{a} \times \exp \left(-K_{0} \times \mathrm{t}\right)+\mathrm{b} \times \exp \left(-K_{1} \times \mathrm{t}\right)(6)$ & $($ Henderson, 1974) \\
\hline
\end{tabular}

$\mathrm{O}$ grau de ajuste de cada modelo considerou a magnitude do coeficiente de determinação $\left(\mathrm{R}^{2}\right)$, o erro médio relativo $(\mathrm{P})$ foi calculado através da equação 7 e o erro médio estimado (SE), conforme a equação 8 .

$$
\begin{aligned}
& \mathrm{P}=\frac{100}{\mathrm{~N}} \sum \frac{\left|\mathrm{Y}-\mathrm{Y}^{\prime}\right|}{\mathrm{Y}} \\
& S E=\sqrt{\frac{\sum\left(Y-Y^{\prime}\right)^{2}}{G L R}}
\end{aligned}
$$

Na qual, Y é o valor observado experimentalmente, Yâ€ $€^{T M}$ é o valor calculado pelo modelo e GLR representa os graus de liberdade do modelo, medido pela diferença entre o número de observações $(\mathrm{N})$ e o número de parâmetros do modelo. 


\section{RESULTADOS E DISCUSSÃO}

A Figura 1A é composta pelas curvas dos experimentos realizados na temperatura de 78 ${ }^{\circ} \mathrm{C}$ e velocidade do ar de $0,5 \mathrm{~m} / \mathrm{s}$ (experimentos 2, 4 e 6), na Figura $1 \mathrm{~B}$ são exibidas as curvas obtidas a temperatura de $68{ }^{\circ} \mathrm{C}$ e velocidade do ar a $1 \mathrm{~m} / \mathrm{s}$ (experimentos 1,3 e 5 ). As maiores temperaturas mostrou ter maior influência na secagem quando comparada as velocidades do ar provocando curvas mais acentuadas, ou seja, uma redução no tempo para atingir o equilíbrio.

Figura 1 - Curvas experimentais de secagem. A) Temperatura a $78^{\circ} \mathrm{C}$ e velocidade de $0,5 \mathrm{~m} / \mathrm{s}$ e B) Temperatura a $68^{\circ} \mathrm{C}$ e velocidade de $1 \mathrm{~m} / \mathrm{s}$.

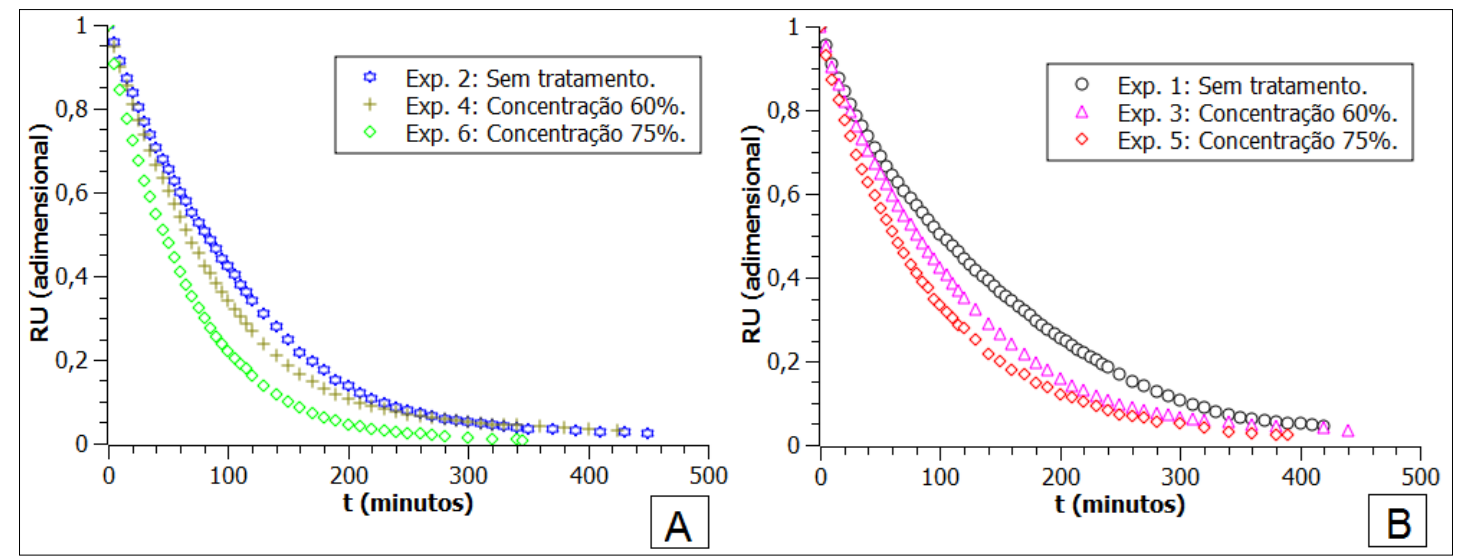

Os menores valores de razão de umidade em função do tempo foram para as curvas 5 e 6, nas quais, a banana sofreu desidratação osmótica prévia em solução de sacarose a $75 \%$ $(\mathrm{m} / \mathrm{m})$ e os maiores valores de razão de umidade em função do tempo estão presentes nas curvas 1 e 2, onde a banana não sofreu tratamento osmótico. Valores intermediários foram encontrados para o experimento 3 e 4, sendo que nesses a amostra sofreu desidratação osmótica antes da secagem convectiva em solução de sacarose na concentração de $60 \%$ $(\mathrm{m} / \mathrm{m})$. Como a temperatura e a velocidade do ar são constantes em cada gráfico, presume-se que a desidratação osmótica foi eficaz na retirada de conteúdo de água, conforme o encontrado por Kotovicz (2011).

$\mathrm{Na}$ Tabela 3 são apresentados os coeficientes de determinação $\left(\mathrm{R}^{2}\right)$, os erros médios relativos $(\mathrm{P})$ e os erros médios estimados (SE), para cada um dos modelos matemáticos avaliados.

Tabela 3 - Coeficientes de determinação, erros médios relativos e erros médios estimados

\begin{tabular}{c|l|l|l|l|l|l|c}
\hline \multicolumn{2}{c|}{ Experimento } & \multicolumn{1}{|c|}{$\mathbf{1}$} & $\mathbf{2}$ & $\mathbf{3}$ & $\mathbf{4}$ & $\mathbf{5}$ & $\mathbf{6}$ \\
\hline \multirow{4}{*}{ Newton } & $\mathrm{R}^{2}(\%)$ & 99,713 & 99,694 & 99,896 & 99,843 & 99,788 & 99,944 \\
\cline { 2 - 8 } & $\mathrm{SE} \mathrm{( \% )}$ & 0,1519 & 0,1945 & 0,1152 & 0,1311 & 0,1595 & 0,0659 \\
\cline { 2 - 8 } & $\mathrm{P}(\%)$ & 7,0669 & 11,7527 & 5,9849 & 11,759 & 8,5968 & 5,7896 \\
\hline \multirow{4}{*}{ Page } & $\mathrm{R}^{2}(\%)$ & 99,717 & 99,880 & 99,904 & 99,862 & 99,986 & 99,951 \\
\cline { 2 - 8 } & $\mathrm{SE} \mathrm{( \% )}$ & 0,1529 & 0,1273 & 0,1095 & 0,1172 & 0,0398 & 0,0691 \\
\cline { 2 - 8 } & $\mathrm{P}(\%)$ & 7,6066 & 8,1351 & 5,6608 & 11,668 & 1,8443 & 5,1509 \\
\hline Midilli e & $\mathrm{R}^{2}(\%)$ & 99,916 & 99,916 & 99,934 & 99,975 & 99,987 & 99,965 \\
\hline
\end{tabular}




\begin{tabular}{|c|c|c|c|c|c|c|c|}
\hline \multirow[t]{2}{*}{ Kucuk } & SE (\%) & 0,0802 & 0,1043 & 0,0904 & 0,0486 & 0,0391 & 0,0607 \\
\hline & $\mathrm{P}(\%)$ & 4,5874 & 5,1869 & 3,8877 & 2,3453 & 1,8293 & 4,5679 \\
\hline \multirow{3}{*}{$\begin{array}{l}\text { Aproximaçã } \\
\text { o da difusão }\end{array}$} & $\mathrm{R}^{2}(\%)$ & 99,775 & 99,911 & 99,897 & 99,872 & 99,985 & 99,971 \\
\hline & SE $(\%)$ & 0,1343 & 0,1111 & 0,1173 & 0,1118 & 0,0431 & 0,0583 \\
\hline & $\mathrm{P}(\%)$ & 6,4993 & 8,4706 & 6,0519 & 11,296 & 2,9176 & 5,3953 \\
\hline \multirow{3}{*}{$\begin{array}{c}\text { Henderson e } \\
\text { Pabis }\end{array}$} & $\mathrm{R}^{2}(\%)$ & 99,766 & 99,743 & 99,897 & 99,856 & 99,918 & 99,964 \\
\hline & SE $(\%)$ & 0,1386 & 0,1871 & 0,1165 & 0,1273 & 0,0908 & 0,0637 \\
\hline & $\mathrm{P}(\%)$ & 7,7325 & 10,4756 & 6,0449 & 10,904 & 5,4689 & 5,4822 \\
\hline \multirow{3}{*}{ Dois termos } & $\mathrm{R}^{2}(\%)$ & 99,766 & 99,743 & 99,897 & 99,856 & 99,985 & 99,964 \\
\hline & SE $(\%)$ & 0,1408 & 0,1909 & 0,1190 & 0,1331 & 0,0439 & 0,0652 \\
\hline & $\mathrm{P}(\%)$ & 7,7328 & 10,4757 & 6,0459 & 10,985 & 2,8289 & 5,4819 \\
\hline
\end{tabular}

Os modelos matemáticos obtiveram elevados valores de $\mathrm{R}^{2}$, o que, segundo Madamba et al. (1996) significa um bom ajuste para representação de secagem. O modelo que obteve os menores erros relativos foi o de Midilli e Kucuk, em seguida foi o de Page. Para os erros médios estimados o modelo de Midilli e Kucuk também apresentou os menores valores, sendo assim, o modelo que melhor representou a secagem. Os coeficientes desse modelo são apresentados na Tabela 4.

Tabela 4 - Valores das constantes e dos coeficientes do modelo de Midilli e Kucuk

\begin{tabular}{c|c|c|c|c|c|c|c}
\hline \multicolumn{2}{c|}{ Experimento } & $\mathbf{1}$ & $\mathbf{2}$ & $\mathbf{3}$ & $\mathbf{4}$ & $\mathbf{5}$ & $\mathbf{6}$ \\
\hline \multirow{3}{*}{$\begin{array}{c}\text { Midilli e } \\
\text { Kucuk }\end{array}$} & $\mathrm{K}$ & 0,00864 & 0,00448 & 0,00617 & 0,00686 & 0,014757 & 0,014194 \\
\cline { 2 - 8 } & $\mathrm{n}$ & 0,93842 & 1,14378 & 1,07165 & 1,10061 & 0,934075 & 1,010803 \\
\cline { 2 - 8 } & $\mathrm{a}$ & 0,98503 & 0,97460 & 0,97660 & 0,98677 & 0,994854 & 0,982772 \\
\cline { 2 - 8 } & $\mathrm{b}$ & 0,00013 & 0,00002 & 0,00003 & 0,00007 & 0,000001 & 0,000003 \\
\hline
\end{tabular}

\section{CONCLUSÃO}

As curvas de secagem sugerem que o pré-tratamento com desidratação osmótica favorece a secagem global, diminuindo o tempo de secagem, e que quanto mais concentrada a solução desidratante maior é a saída de água, assim, esse procedimento é útil para preservar as características sensoriais do produto final e auxiliar na secagem.

Os experimentos feitos sob maior temperatura apresentaram curvas mais acentuadas do que os que foram realizados sob temperatura menor quando as outras variáveis estavam constantes, indicando que temperaturas maiores promovem maior transferência de massa de água da amostra para o ar, diminuindo o tempo necessário para se atingir baixas umidades.

Os modelos matemáticos podem ser úteis para predizer o comportamento da secagem de bananas nas condições analisadas, pois todos obtiveram elevados valores de coeficiente de determinação, sendo que o melhor ajuste foi encontrado usando o modelo de Midilli e Kucuk.

\section{REFERENCIAS}

CANO-CHAUCA, M.; RAMOS, A. M.; STRINGHETA, P. C.; MARQUES, J. A.; SILVA,

P. I. Curvas de secagem e avaliação da atividade de água da banana passa. B. CEPPA, Curitiba, v. 22, n.1, p 121-132, 2004. 
CORRÊA, J. L. G.; SILVA, E. D.; BATISTA, M. B.; AROLA, F.; FIOREZE, R. Desidratação osmótica de tomate seguida de secagem. Rev. Bras. de Produtos Agroindustriais, Campina Grande, v.10, n.1, p.35-42, 2008.

GOMES, A. T.; CEREDA, M. P.; VILPOUX, O. Desidratação Osmótica: uma tecnologia de baixo custo para o desenvolvimento da agricultura familiar. Rev. Brasileira de Gestão e Desenvolvimento Regional, São Paulo, v. 3, n. 3, p. 212-226, 2007.

HENDERSON, S. M. Progress in developing the thin layer drying equation. Transactions of the $A S A E$, v. 17, p. 1167-1168, 1974.

HENDERSON, S. M., PABIS, S. Grain drying theory: temperature effect on drying coefficient. Journal of Agricultural Engineering Research, v. 06, p. 169-174, 1961.

KASSEM, A. S. Comparative studies on thin layer drying models for wheat. In: International Congress On Agricultural Engineering, 13th, Morocco, v. 06, 1998.

KOTOVICZ, V. Otimização da desidratação osmótica e secagem do yacon. 2011. Dissertação (Mestrado em Tecnologia de Alimentos) - Universidade Federal do Paraná. Curitiba, 2011.

LEWIS, W. K. The drying of solid materials. The Journal of Industrial and Engineering Chemistry, v. 13, n. 05, p.427-433, 1921

MADAMBA, P. S.; DRISCOLL, R. H.; BUCKLE, K. A. Thin layer drying characteristics of garlic slices. Journal of Food Engineering, v.29, n.1, p.75-97, 1996.

MARQUES, L. F.; DUARTE, M. E. M.; MATA, E. R. M. C.; NUNES, L. S.; COSTA, T. L.; COSTA, P. B. S.; DUARTE, S. T. G. Secagem precedida de desidratação osmótica de pseudofruto de caju: comparação entre modelos matemáticos aplicados. Rev. Brasileira de Produtos Agroindustriais. Campina Grande, v. 9, n. 2, p.170, 2007.

PAGE, G. E. Factors influencing the maximum rates of air drying shelled corn in thin layers. West Lafayette: Purdue University, 1949.

QUEIROZ, V. A. V.; BERBERT, P. A.; MOLINA, M. A. B.; GRAVINA, G. A.; QUEIROZ, L. R.; DELIZA, R. Desidratação por imersão-impregnação e secagem por convecção de goiaba. Pesq. agropec. bras., Brasília, v.42, n.10, p.1479-1486, 2007.

SOUZA NETO, M. A.; MAIA, G. A.; LIMA, J. R.; FIGUEIREDO, R. W.; SOUZA, M. S. M.; LIMA, A. S. Desidratação osmótica de manga seguida de secagem convencional: avaliação das variáveis de processo. Ciênc. agrotec., Lavras, v. 29, n. 5, p. 1021-1028, 2005.

MIDILLI, A.; KUCUK, H.; YAPAR, Z. A New model for single layer drying. Drying Technology, v. 20, n. 07, p. 1503-1513, 2002. 